EPJ Web of Conferences 61, 03003 (2013)

DOI: $10.1051 /$ epjconf/ 20136103003

(C) Owned by the authors, published by EDP Sciences, 2013

\title{
Discovery of synchrotron emission from a YSO jet
}

\author{
Carlos Carrasco-González ${ }^{1,2, a}$, Luis F. Rodríguez² ${ }^{2}$ Guillem Anglada ${ }^{3}$, Josep Martí ${ }^{4}$, Jose M. Torrelles ${ }^{5}$, and Mayra \\ Osorio $^{3}$ \\ ${ }^{1}$ Max-Planck-Institute für Radioastronomie (MPIfR, Germany) \\ ${ }^{2}$ Centro de Radioastronomía y Astrofísica (CRyA, Mexico) \\ ${ }^{3}$ Instituto de Astrofísica de Andalucía (IAA, Spain) \\ ${ }^{4}$ Departamento de Física, Escuela Politécnica Superior de Jaén (EPSJ), Universidad de Jaén (Spain) \\ ${ }^{5}$ Instituto de Ciencias del Espacio (CSIC/IEEC)-UB (Spain)
}

\begin{abstract}
Synchrotron emission at radio wavelengths is commonly found in relativistic jets from active galactic nuclei (AGNs) and microquasars and allows the study of the magnetic field in these kind of jets. In contrast, the radio emission from jets from young stellar objecs (YSOs) is usually of very different nature: thermal free-free emission, which does not provide direct information about their magnetic field. Thus, that the magnetic field is still one of the most unknown physical parameters in these YSO jets. However, very recently, we detected for the first time polarized synchrotron emission from of a YSO (HH 80-81), a result that has two important consequences. First, it allows to study the magnetic field in a YSO jet by analyzing the properties of the synchrotron emission, in a similar way than in the case of AGN jets. Secondly, the presence of synchrotron emission in a YSO jet implies the presence of relativistic particles, and therefore, an acceleration mechanism that should be taken place in these "slow" jets. These results open new windows for the study of YSO jets in a wide range of wavelengths, from radio to $\mathrm{X}$ - and Gamma-rays.
\end{abstract}

\section{Introduction}

Supersonic jets are a ubiquitous phenomenon in the Universe. They are observed to emerge from a wide variety of astrophysical systems: young stellar objects (YSOs), evolved stars, microquasars, and active galactic nuclei (AGNs). Despite their different physical scales (from hundreds to billions of AU), they have strong morphological similarities [1], and a common feature of these systems is the presence of an accretion disk around a central object (a black hole, star or protostar). Theoretical models for the formation of jets assume that the initial acceleration takes place relatively close to the central object as gas from the accretion disk is lifted and centrifugally accelerated along magnetic field lines. This mechanism was first suggested as the origin of jets from accretion disks around black holes (e.g., $[2,3]$ ) and was soon after invoked as the mechanism for YSO jets $[4,5]$. The collimation of the outflow into a jet is though to occur at larger distances from the central object and is also believed from theoretical arguments to be magnetically driven. The magnetic field lines anchored in the rotating accretion disk are proposed to wrap in a helical configuration that is needed to provide the magnetic pressure to collimate the jet via "hoop" stresses [6, 7].

This theoretical picture is well supported by observations in the case of AGN jets, but not in YSO jets. Jets from AGNs have been mapped in exquisite detail by radio interferometers such as the Very Large Array (VLA) (e.g., [8]). Since they radiate primarily via synchrotron emission, the intensity and polarization of this emission can be used to estimate the strength and direction, respectively, of the magnetic field [9]. However, in the case of YSO jets we have a very different situation. A lot information about the physical conditions in YSO jets can be obtained from observations at very different wavelengths. This is not only because they are relatively nearby and thus can be explored with high spatial resolution, but also because they produce narrow emission lines from which fundamental parameters such as the density, temperature, and ionization fraction in the jet can be determined (e.g., [10]). The radial velocity of the jet can also be measured from these lines, and combining this information with images taken over a few years, the full three-dimensional velocity of the jet can be ascertained. The accretion disk, which is intimately related to the jet phenomenon, can also be observed at millimeter and submillimeter wavelengths and important physical parameters, such as the accretion rate, can be obtained from modeling of the spectrum at these wavelengths (e.g., [11, 12]). However, the one missing ingredient for obtaining a complete physical description of jets from YSOs is their magnetic field, ironically perhaps the only parameter that is well determined in the case of AGN jets.

\footnotetext{
ae-mail: c.carrasco@crya.unam.mx
} 


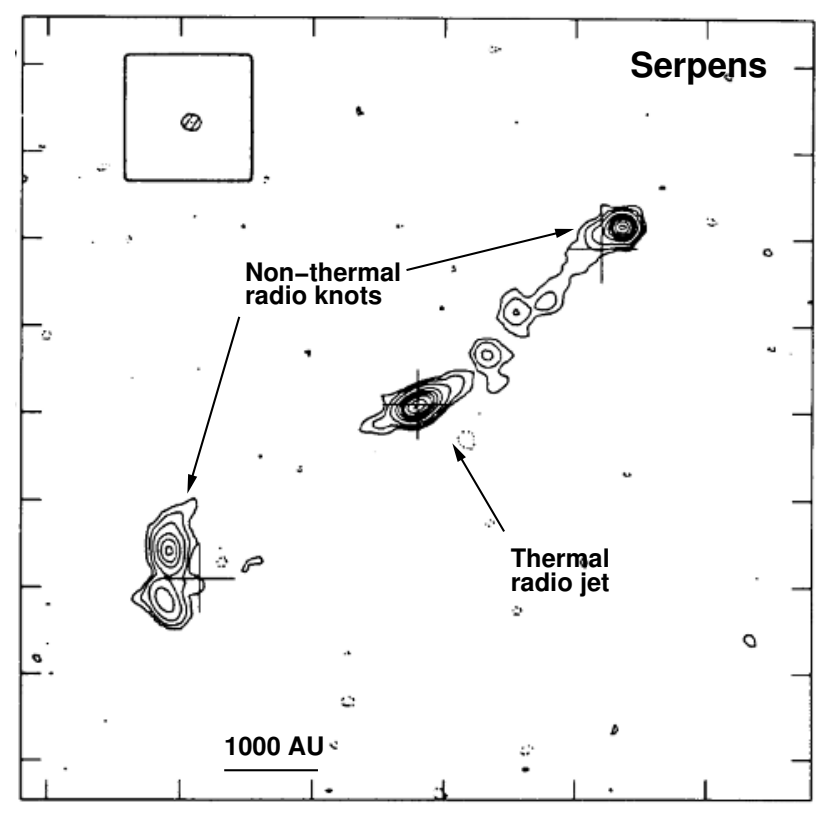

Figure 1. The radio jet in the Serpens star-forming region is one of the first YSO jets where synchrotron emission was proposed as the emission mechanism in strong shocks of the jet with the ambient medium $[13,14]$. The central radio source shows a positive spectral index at radio wavelengths, which is consistent with thermal free-free emission from ionized gas. The two bright sources at the termination points of the radio jet show negative spectral indices indicative of non-thermal emission.

\section{Synchrotron emission in YSO jets}

Jets from YSOs have been long studied at radio wavelengths (e.g., [15-17]). As the material from the jet is launched, it becomes partially ionized due to shocks with the ambient medium and/or internal shocks. Freefree interactions between thermal electrons in this ionized jet produce detectable continuum emission at centimeter wavelengths with a characteristic positive spectral index (the intensity of the emission increases with the frequency). High angular resolution observations at radio wavelength allow the jet material to be traced up to a few hundreds of AU from the protostar. This is the only way to study the base of the pc-scale jets, which cannot be observed at optical or IR wavelengths due to the large extinction near the protostar. These radio sources are called thermal radio jets, in contrast to the radio jets usually observed from AGNs whose main emission mechanism at radio wavelengths is of non-thermal nature (optically thin synchrotron emission) and show very different characteristics: negative spectral indices at $\mathrm{cm}$ wavelengths, and linear polarization from which information about the orientation and strength of the magnetic field can be obtained.

In the last two decades, non-thermal emission has been also proposed to be present in several YSO jets (e.g., $[13,18-21])$. This non-thermal emission is usually found in strong radio knots showing negative spectral indices at centimeter wavelengths (see Fig. 1). These non-thermal radio knots usually appear in pairs, moving away from the central protostar at velocities of several hundreds of kilometers per second. Because of these characteristics, it has been proposed that these knots would be tracing strong shocks of the jet against dense material in the surrounding molecular cloud where the protostar is forming. Their non-thermal nature has been interpreted as synchrotron emission from a small population of relativistic particles that would be accelerated in the ensuing strong shocks.

This scenario has been confirmed only very recently, after we presented the detection of linearly polarized emission from a YSO jet [22] (see Fig. 2). This result provided for the first time conclusive evidence for the presence of synchrotron emission in a jet from a YSO and allowed us to directly measure and study the properties of its magnetic field (strength and morphology). Interestingly, we found that the characteristics of the synchrotron emission are consistent with a helical configuration of the magnetic field: the magnetic field appears parallel to the jet direction and the polarization degree increase to the edges of the jet (see Fig. 2). This is similar to what is commonly found in AGN jets (e.g. [23]), and strongly suggests that there is a universal mechanism that might be operating in all astrophysical jets.

Our discovery suggests that mapping of linear polarization in a set of YSO jets in combination with detailed theoretical modeling may lead to a deeper understanding of the overall jet phenomenon. Radio synchrotron emission in YSO jets seems to be intrinsically much weaker and difficult to study than in AGN jets. So far, non-thermal emission has been identified in a handful of YSO jets through measurements of their spectral indices, but only one case (HH 80-81, one of the brightest and most powerful YSO jets known) has been observed with enough sensitivity to detect and study its linear polarization (which is only a fraction of the total continuum emission). The new generation of ultrasensitive radio interferometers, such as the Jansky VLA (JVLA), offers now the possibility to perform new observations with an unprecedent high sensitivity, allowing a detailed study of the magnetic field in a sample of YSO jets.

Detection of linearly polarized emission at several wavelengths would allow to infer the properties of the magnetic field in a similar way than is commonly performed in AGN jets. The magnetic field strength can be estimated from the spectral energy distribution at $\mathrm{cm}$ wavelengths (e.g., [26, 27]), while the magnetic field morphology can be obtained from the properties of the linear polarization (polarization angle, polarization degree and Faraday rotation). For non-relativistic jets, the apparent magnetic field (magnetic field averaged along the line-ofsight) is perpendicular to the direction of the linear polarization. Moreover, theoretical models of helical magnetic fields predict gradients of the polarization degree and Faraday rotation measurements along and across the jet [28]. Thus, by comparing the observational results with theoretical models, the 3-D morphology of the magnetic field can be inferred.

At present, we are using the JVLA to observe a small sample of YSO jets at several wavelengths. Although the 


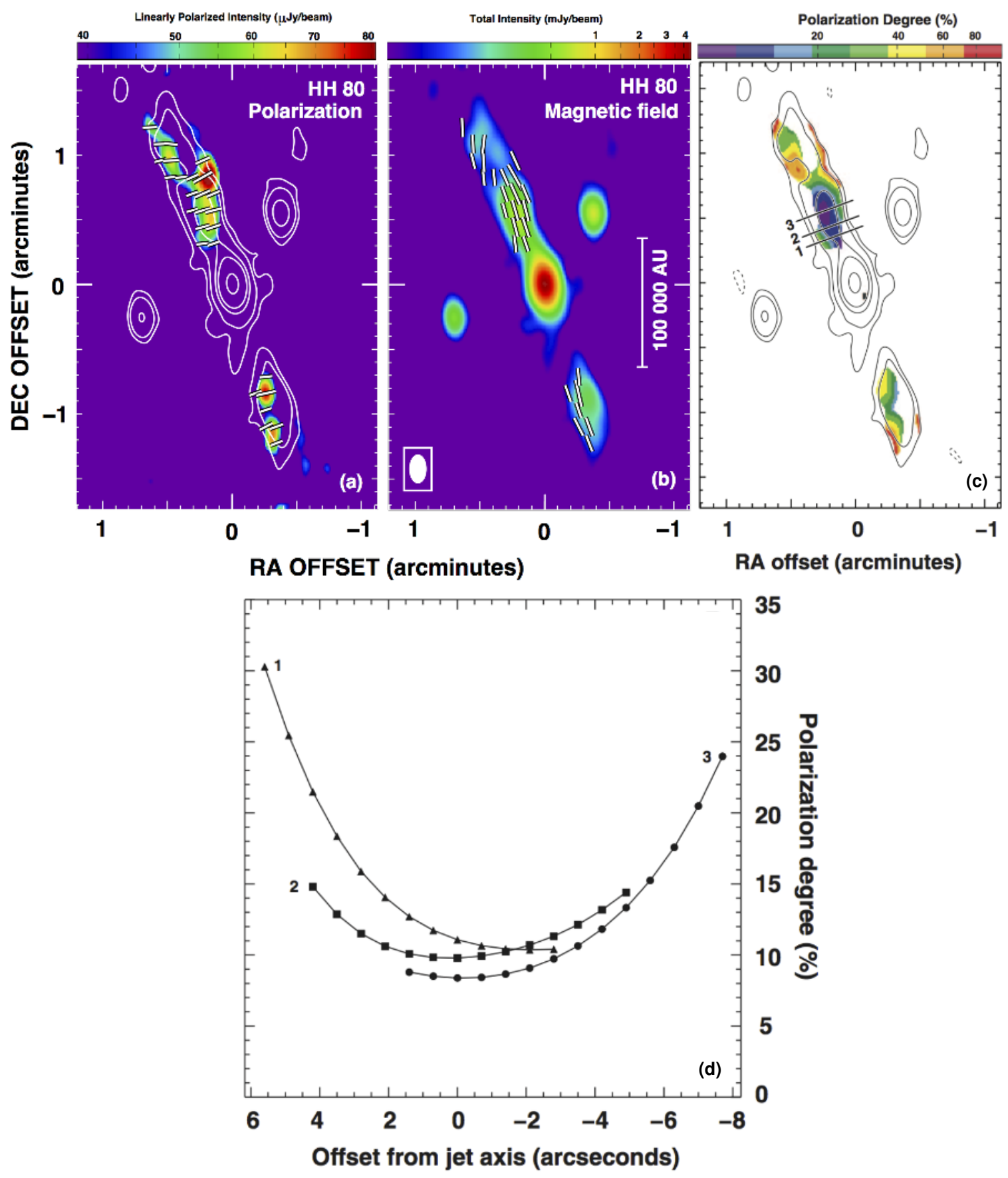

Figure 2. Detection of linearly polarized emission at radio wavelengths in the YSO jet HH 80-81 (from [22]). In panel (b) the intensity of the polarized emission is shown in colors while the total continuum emission is shown in contours. Polarized emission is detected in the northern and southern lobes, where non-thermal radio emission was previously proposed [19]. These results provided for the first time conclusive evidence of the presence of synchrotron emission in a jet from a YSO and allowed to directly study the magnetic field. In panel (c) we show a polarization degree map and in panel (d) the polarization degree along three slices transversal to the jet direction. As can be seen, the polarization degree increases across the jet width, which is consistent with a helical magnetic field.

full final capabilities of the JVLA were not yet available at the time of these observations, our preliminary results are already very exciting. A new radio map of the $\mathrm{HH} 80-81$ jet with higher angular resolution and sensitivity revealed a morphology much more similar to AGN jets: a highly collimated radio jet ending in two extended radio lobes (see Figure 3). A spectral index map shows that both features, the collimated jet and the extended radio lobes are domi- nated by non-thermal emission. This result suggests that this kind of observations will help reveal synchrotron features in YSO jets which had remained hidden in previous, less sensitive, radio observations. 

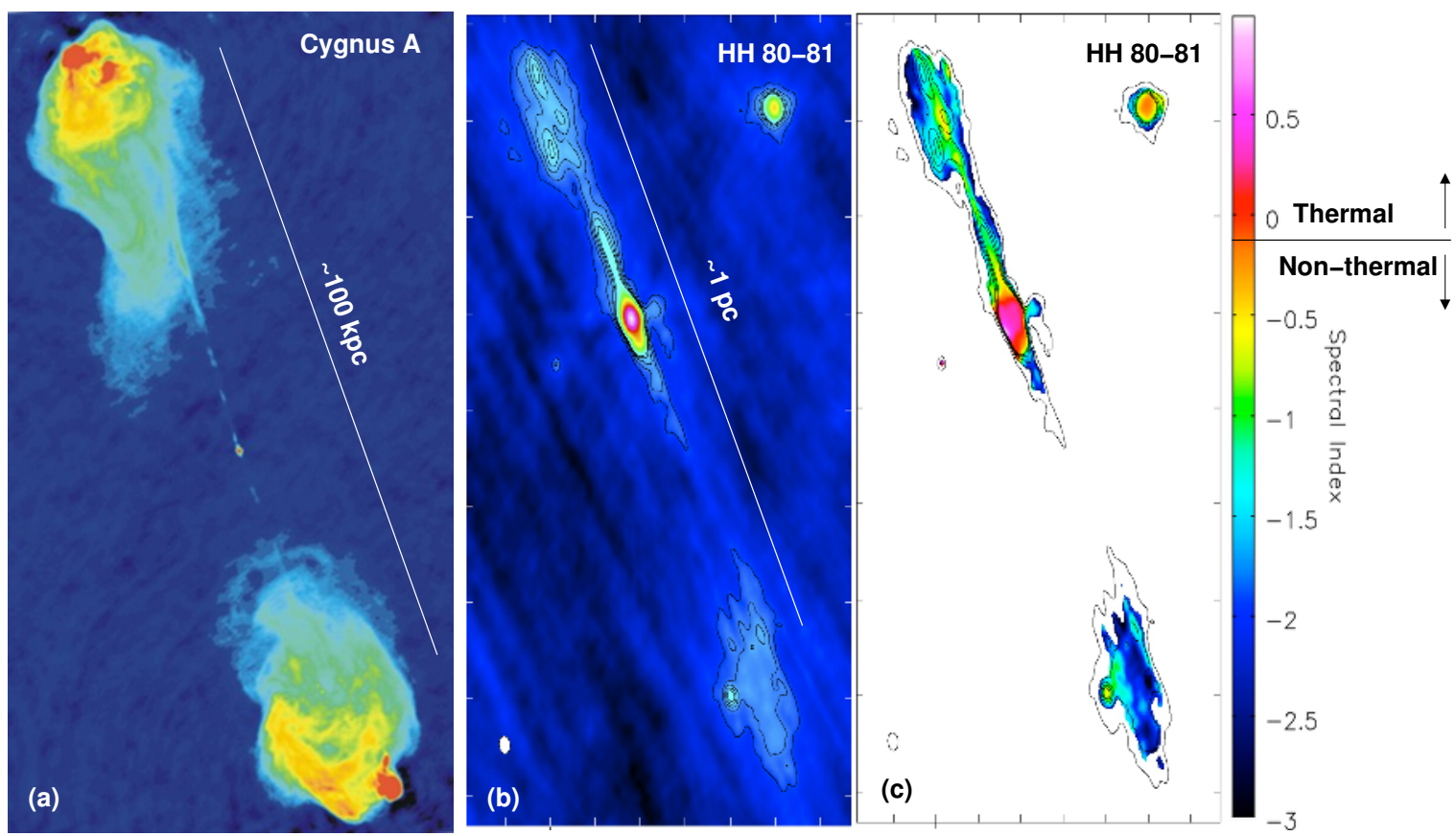

Figure 3. (a): Radio map of the AGN jet in Cygnus A (from [24]). (b and c): New JVLA radio observations of the YSO jet HH 80-81 [25]. The unprecedent combination of high sensitivity and high angular resolution of the JVLA revealed a morphology of the radio jet very similar to what is commonly found in AGN jets: a highly collimated jet ending in two extended radio lobes. A spectral index map (panel c) shows that the emission mechanism in both the extended lobes and the collimated jet is of non-thermal nature. The differences in the negative spectral indices of the lobes are most probably related to differences in the cooling time of the relativistic particles.

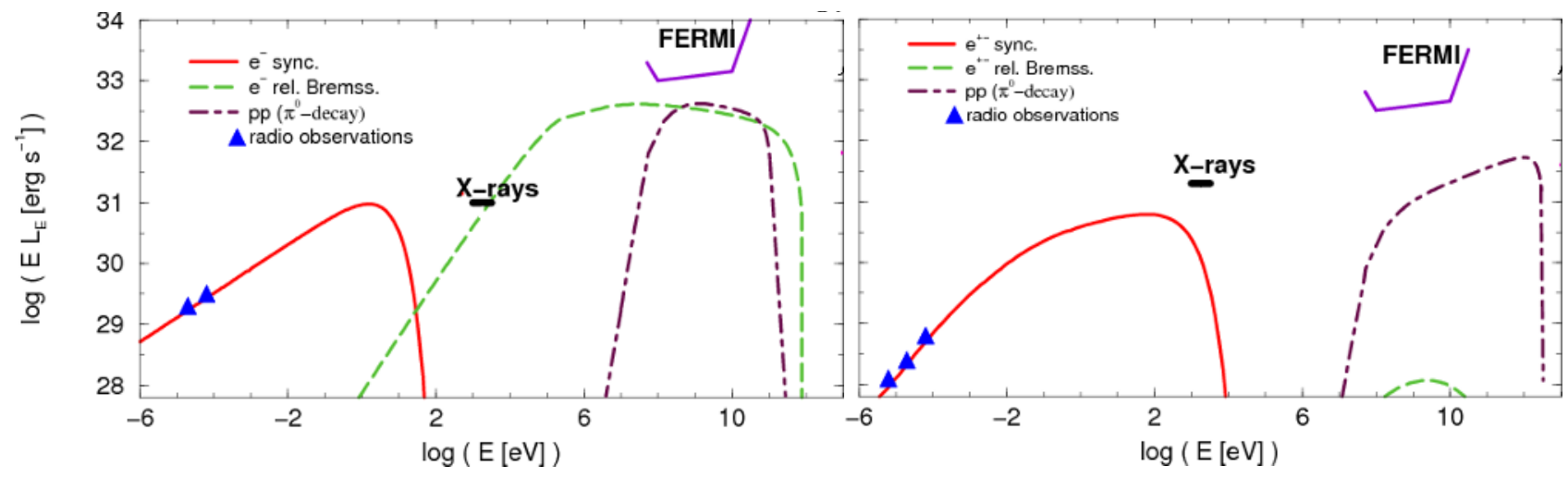

Figure 4. Spectral energy distributions of the non-thermal emission in YSO jets for different scenarios (from [33]). The left panel shows a case in which the radio emitting particles are primary electrons accelerated in the shock, while the right panel shows a case for secondary electrons and positrons from pp collisions (see [33] for details). The $1 \mathrm{yr} / 5 \sigma$ sensitivity of Fermi in the direction of the galactic plane is shown. Note that, in each scenario, different emission mechanisms are expected to dominate in the X-and gamma-ray regimes.

\section{Particle acceleration and high-energy phenomena}

The discovery of synchrotron emission from YSO jets also implies that high-energy phenomena should be taking place in these objects. As commented above, the synchrotron emission seems to be produced mainly where the jet strongly impacts with dense material of the surrounding molecular cloud. In this situation, particles like electrons and protons gain energy by diffusing upstream across a shock front [29]. The shock then catches up with the particle, but it diffuses across it again, gaining even more energy as it does so. This process, known as diffusive shock acceleration (DSA), can cause particles moving at a few hundreds of kilometers per second to reach velocities near the speed of light. The DSA mechanism is already known 


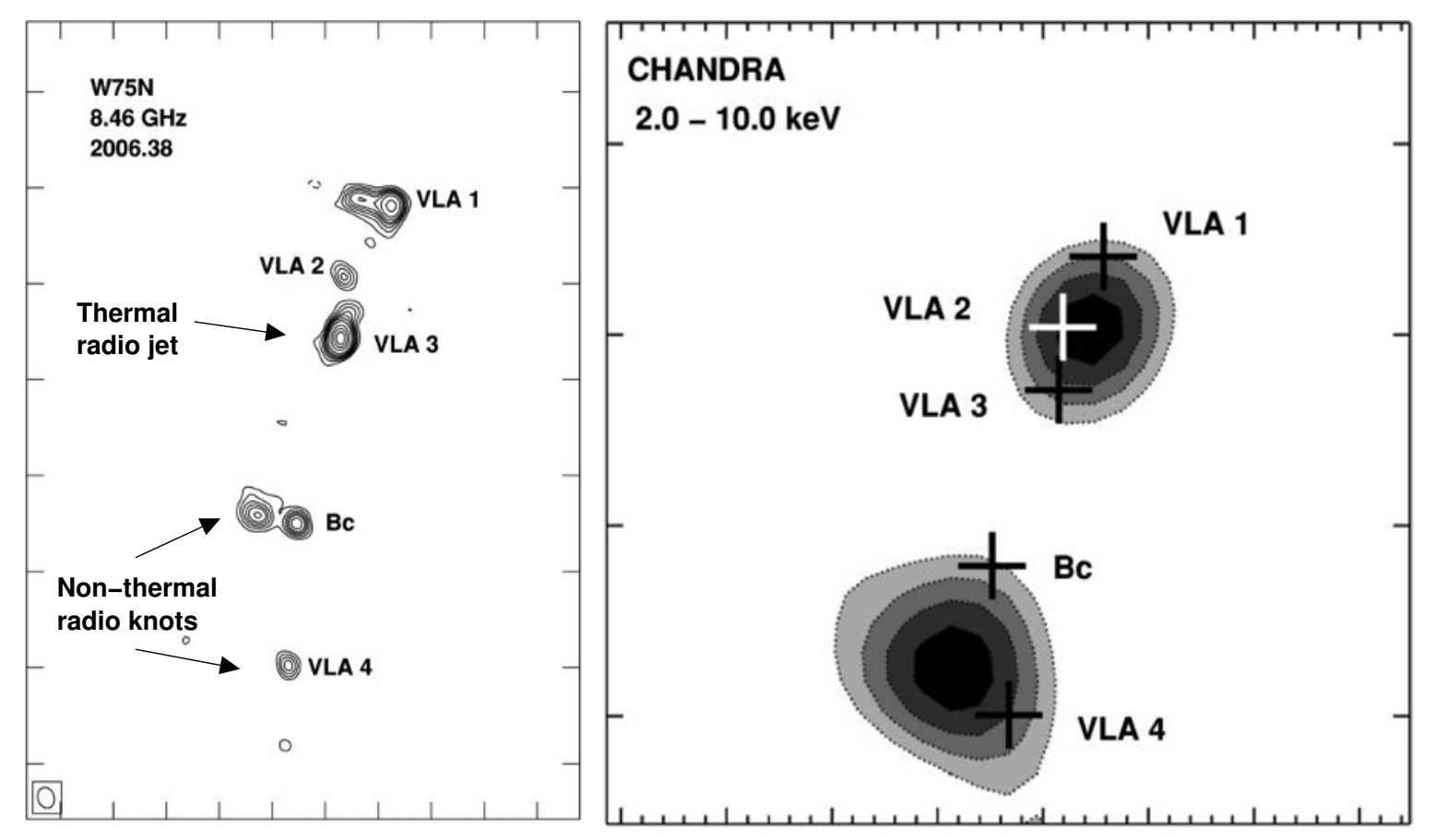

Figure 5. Radio VLA map (left) and CHANDRA X-ray image (right) of the W75N star-forming region (from [36]). Source VLA 3 is associated with a YSO which is driving a jet in the NE-SW direction. The radio knots Bc and VLA 4 show proper motions of several hundreds of kilometers per second to the SW and their radio emission is of non-thermal nature. X-Ray emission is detected associated with the non-thermal knots Bc and VLA 4, most probably associated with a strong shock of the jet with the ambient medium. However, the low signal-to-noise ratio of the observations does not allow us to discern which is the emission mechanism at X-rays.

to work in jets from AGNs (e.g., [30]), supernova remnants (e.g., [31]) and colliding wind binaries (e.g., [32]), but in those cases the shocks are produced by the collision of material with velocities of at least several thousand kilometers per second. The material in YSO jets is much slower, at most a few hundred kilometers per second, but the new findings suggest that DSA can operate even in these puny jets. Thus, jets from young stars could be a new extreme testing ground for the theory of how cosmic rays are made.

A population of relativistic electrons in the complex environment of the massive molecular cloud in which the protostar is being formed will produce high-energy radiation through a variety of processes: inverse Compton (IC) scattering of infrared (IR) photons from the cloud, relativistic bremsstrahlung, and, if protons are accelerated as well, inelastic proton-proton (pp) collisions. Theoretical models of shocks in YSO jets predict that all these processes will produce detectable emission in a broad wavelength range, from radio to gamma-rays (e.g., [33, 34]; see Fig. 3). Different emission mechanisms are expected at X-and gamma-rays depending on the efficiency of the particle acceleration mechanism, the type of particles that are accelerated, the strength of the magnetic field, and the physical conditions in the shock (see Fig. 3). Therefore, by combining radio, X-Ray and gamma-ray observations, valuable information about the conditions in YSO jets can be obtained.

In the last years, observations with XMM Newton and CHANDRA have allowed to detect X-ray emission asso- ciated with several YSO jets (e.g., [35-37]). Interestingly, all YSO jets showing radio synchrotron emission are also associated with X-ray emission, suggesting that emission mechanism at both wavelengths could be related. A good example is the W75N-VLA 3 jet where, using CHANDRA archive data, we detected hard X-ray emission associated with the non-thermal shocks in the jet [36] (see Fig. 4). However, the X-ray emission in YSO jets is usually very weak, and in most cases the low sensitivity of the observations has not allowed to study the X-ray spectra in detail to discern which is the emission mechanism. Higher sensitive X-Ray observations are needed in this kind of objects in order to extract valuable information about the high-energy processes in the shocks of YSO jets.

Finally, although it is not expected that massive protostars to be among the brightest sources detected by Fermi, the theoretical models predict that they could show up in further analysis of weaker sources after a few years of observation [33]. This would open a new window to star formation studies [38].

\section{References}

[1] De Young, Science 252, 389 (1991)

[2] Blandford \& Payne, MNRAS, 199, 883 (1982)

[3] McKinney \& Blandford, MNRAS, 394, 126 (2009)

[4] Pudritz \& Norman, ApJ, 274, 677 (1983)

[5] Shu et al., ApJ, 429, 781 (1994)

[6] Livio, ASPC, 121, 845 (1997)

[7] Pudritz et al., PPV, 277 (2007) 
[8] Sparsky et al., ApJ, 473, 254 (1996)

[9] Dulwich et al., MNRAS, 374, 1216 (2007)

[10] Ray et al., PPV, 321 (2007)

[11] Carrasco-González et al., ApJ, 746, 71 (2012)

[12] Carrasco-González et al., ApJ, 752, 29 (2012)

[13] Rodríguez et al., ApJ, 346, L85 (1989)

[14] Curiel et al., ApJ, 415, 191 (1993)

[15] Rodríguez, RMxAC, 1, 1 (1995)

[16] Rodríguez, RMxAC, 4, 7 (1996)

[17] Anglada, ASPC, 93, 3 (1996)

[18] Rodríguez et al., ApJ, 626, 953 (2005)

[19] Martí et al., ApJ, 416, 208 (1993)

[20] Garay et al., ApJ, 459, 193 (1996)

[21] Wilner et al., ApJ, 513, 775 (1999)

[22] Carrasco-González et al., Science 330, 1209 (2010)

[23] Gómez et al., ApJ, 681, L69 (2008)
[24] Perley et al., ApJ, 285, 35 (1984)

[25] Carrasco-González et al., in preparation

[26] Pacholczyk, Radio Astrophysics (1970)

[27] Beck \& Krause, AN, 326, 414 (2005)

[28] Lyutikov et al., MNRAS, 360, 869 (2005)

[29] Drury, MNRAS, 251, 340 (1991)

[30] Blandford et al., Scientific American, 246, 124 (1982)

[31] Castro et al., ApJ, 717, 372 (2010)

[32] de Becker, A\&ARv, 14, 171 (2007)

[33] Bosch-Ramon et al., A\&A, 511, 8 (2010)

[34] Araudo et al., A\&A, 476, 1289 (2007)

[35] Skinner et al., ApJ 658, 1144 (2007)

[36] Carrasco-González et al., AJ, 139, 2433 (2010)

[37] Anderson et al., AJ 142, 158 (2011)

[38] Romero, AIPC, 1085, 97 (2008). 